\title{
Aroma Acupoint Therapy for Symptom Management with Adolescent Patients: Early Experiences from School-Based Health Centers
}

\author{
Avital M. Fischer, MD, ${ }^{1,2}$ Peter Holmes, LAc, MH, ${ }^{3}$ Yasmin Z. Bahar, DNP, FNP-BC, ${ }^{4}$ \\ Susan Vacca, PhD, NP, Sarah Goldberg, NP, and Melanie A. Gold, DO, ${ }^{4} \mathrm{MQ}^{1,2,4}$
}

\begin{abstract}
Objectives: The American Academy of Pediatrics has long recognized a need for more resources for integrative medicine for health care providers and for patients. The aim of this study was to introduce Aroma Acupoint Therapy (AAT) - a relatively new integrative medicine modality using essential oils to activate acupoints - and to describe the early experiences of providers using AAT for adolescents at school-based health centers (SBHCs).

Materials and Methods: This was a case series of 15 adolescents treated with 1 of 2 AAT specific protocols plus standard medical therapy between April 2018 and February 2019 at SBHCs. Of these 15 patients, ages 12-19, 14 were female. Clinical characteristics and treatment courses were abstracted by retrospective review of the patients' electronic medical records. The main outcomes and measures for this research were clinical characteristics, treatment courses and pre- and post-treatment pain scores for adolescents treated with AAT.

Results: All 15 patients had nonspecific symptoms, including headaches, menstrual cramps, nausea, shortness of breath, chest pain, back pain, or dizziness. Pain scores were recorded in 8 of 15 encounters, and suggested improvements in most patients. Subjective documentation by the providers also suggested that most of these patients had reduced symptoms.

Conclusions: With the call for nonopiate and integrative approaches to pain management, there is an urgent need to study the effectiveness of such modalities, such as AAT. It is safe, inexpensive, easy to learn, and is well-received by both health care providers and patients.
\end{abstract}

Keywords: essential oil, acupoint, integrative medicine, aroma acupoint therapy (AAT), school-based health centers, adolescent

\section{INTRODUCTION}

$\mathbf{C}$ OMPLEMENTARY AND INTEGRATIVE APPROACHES to medicine are essential for holistic and patient-centered care. They can include nonpharmacologic approaches to pain management, which comprise an urgent current need. ${ }^{1}$
As the demand for integrative medicine modalities increases, there is also an increasing need for reliable literature and resources on these modalities. ${ }^{2,3}$

Aroma Acupoint Therapy (AAT) is a treatment modality first developed in the 1990s by author Peter Holmes, LAc, $\mathrm{MH}$, when he blended his clinical practice in acupuncture

\footnotetext{
${ }^{1}$ Department of Pediatrics, Columbia University Irving Medical Center, New York, NY, USA.

${ }^{2}$ Department of Pediatrics, New York-Presbyterian, New York, NY, USA.

${ }^{3}$ Snow Lotus Seminars, Santa Rosa, CA, USA.

${ }^{4}$ Center for Community Health and Education, School Based Health Centers-New York, New York, NY, USA.
} 
with his passion for aromatherapy, using genuine essential oils designed for therapeutic use. AAT is a safe and gentle treatment that involves placing specific essential oils on specific Chinese acupoints to trigger energetic changes in patients' bodies. ${ }^{4}$ The acupoints are the same as those utilized in acupuncture, with essential oils replacing needles as the stimuli.

Tense and Tense Alternating with Weak are 2 conditions or syndromes defined by Mr. Holmes that include physical as well as psychoemotional attributes. In general, Tense conditions (also called Wired) have the quality of contraction, spasm, increased sensation or pain, irritability, anxiety, nervousness, or restlessness. Tense Alternating with Weak conditions (also called Wired and Tired) are similar to Tense conditions but with elements of fatigue, exhaustion, and/or mood dysregulation. Mr. Holmes developed 2 protocols, 1 each to treat Tense and Tense Alternating with Weak conditions utilizing AAT, which he has found to be clinically safe, often effective after 1 treatment, and easy to teach health care providers and patients.

The primary aim of this case series was to describe the use of AAT for symptom management in adolescent patients presenting to school-based health centers (SBHCs) in New York City. SBHCs are comprehensive primary-care centers functioning in public schools; SBHCs offer a full range of primary-care services at no out-of-pocket costs, regardless of patients' insurance status.

\section{Background}

Practitioners have utilized various preparations of aromatic plants for symptom and disease management as far back as 4500 BCE. $^{5}$ Essential oils themselves were first extracted from aromatic plants by alchemists in the Alexandrian era 2 millennia ago, and then in the fourteenth century in France. These oils became valuable remedies in European pharmacy and were listed in pharmacopeias and dispensatories. French medicine in particular has made consistent use of essential oils in treatment since the 1810s. ${ }^{4}$ Essential oils provide a range of therapeutic benefits for addressing a variety of health concerns through inhalation (aromatherapy), topical application, and ingestion. Symptom categories include mental-health issues, pain, gastrointestinal complaints, and respiratory problems, among many other conditions. $^{4,5}$

The medical literature contains articles on several randomized controlled trials designed to evaluate the therapeutic potential of essential oils in the pediatric population. Some of these studies have shown promising initial results for management of pediatric pain and anxiety, particularly as an adjunct therapy added to standard medical therapy. ${ }^{6-13}$ However, these studies were often limited by small sample sizes and inadequate control groups. Additionally, the literature is predominantly in aromatherapy (inhaled essential oils) rather than topical application of essential oils.
To the current authors' knowledge, there are 2 randomized controlled trials in the literature on adult patients that studied the effects of essential-oil application to an acupoint for treating lower-back pain and chronic neck pain, ${ }^{14,15}$ and no studies published in the literature on pediatric patients. As AAT is a novel therapeutic approach, there is currently no published peer-reviewed literature on AAT's use with adult or pediatric populations.

\section{History of AAT and Proposed Mechanism of Action}

The origins of AAT go back to the early 1990s, when Mr. Holmes began experimenting with using essential oils on acupoints in his acupuncture practice. Finding them very compatible with acupuncture, he gradually developed techniques of using them alone on acupoints, not just in preparation for needling. Over the years, $\mathrm{Mr}$. Holmes also developed the functional diagnostic system of the Six Conditions that became the theoretical backbone of AAT, as well as the model of Fragrance Energetics for tracking the effects of essential oils on individuals. His first seminar on using essential oils in Chinese Medicine was given at the New England School of Acupuncture in 1994.

The theoretical concept proposed by Mr. Holmes is that the natural bioenergetic frequency of an essential oil provides information to an acupoint. In the principle of resonance, the synergy between a certain oil and a particular point can trigger a specific type of therapeutic action in a patient. The key to successful treatment is creating the right synergy between certain oils and certain points. The treatment protocols of AAT reflect decades of Mr. Holmes' experience with synergistic oil-point combinations when performed in a specific sequence.

\section{MATERIALS AND METHODS}

One of the authors (Melanie A. Gold, DO, DMQ), is a pediatrician who is board-certified in adolescent medicine and medical acupuncture, and she is medical director of 7 SBHCs in New York City. She trained in AAT at the Pacific College of Oriental Medicine in this city with Mr. Holmes in April 2017. She then trained SBHC medical providers in the Tense and Tense Alternating with Weak AAT protocols in July 2017 with Annie Canton-Wong, LAc, a classmate in the AAT training. In April 2018, essential oil kits with the four essential oils needed to conduct the Tense and the Tense Alternating with Weak AAT protocols were created and disseminated to the SBHC medical staffs, who were then retrained in the protocol. As the SBHCs medical providers began incorporating AAT into their clinical practices, these practitioners messaged the charts of treated patients securely to Dr. Gold for chart review. 
After approval was granted from the institutional review board of Columbia University Irving Medical Center, a retrospective review was conducted on the electronic medical records of the adolescent patients who were treated with AAT protocols between April 2018 and February 2019. De-identified data were extracted from the medical records of each of these patients, including age, gender, grade, chief complaint, history of present illness, review of systems, physical examination, recorded pain scores and other symptoms prior to and after AAT, other therapies including any pharmacotherapy utilized, and assessment and plan related to the chief complaint.

\section{The Protocols}

The Tense protocol (Fig. 1), also called Calming Therapy, involves placing 1-2 drops of 4 different essential oils on 5 acupoints bilaterally for 90 seconds on each acupoint in the following specific sequence:

(1) Bergamot (Citrus x limon) on the auricular point Shen Men on the triangular fossa of the ear

(2) Lavender (Lavandula angustifolia) on the Large Intestine meridian 4 or LI 4 (Hegu) on the dorsum of the hand between the thumb and index finger

(3) Roman chamomile (Anthemus nobili) on the Liver meridian 6 or LR 3 (Taichong) on the dorsum of the foot between the large and second toes (Roman chamomile is substituted for the original protocol using Blue tansy [Tanacetum anпиит])
(4) Lavender on the Pericardium meridian 6 or PC 6 (Neiguan) on the inner wrist 3 fingers' breadth from the wrist crease between the tendons of the palmaris longus and flexor carpi radialis

(5) Atlas cedarwood (Cedrus atlantica) on the Kidney meridian 3 or KI 3 (Taixi) in the inner leg between the medial malleolus and the Achilles tendon.

The Tense Alternating with Weak protocol (Fig. 2), also called Calming and Strengthening Therapy, is similar to the Tense protocol but involves 3 of these essential oils instead of 4 and is applied on different acupoints in a different sequence. For this protocol, 1-2 drops of essential oil are placed for 90 seconds on each acupoint bilaterally in the following specific sequence:

(1) Bergamot on the auricular point Shen Men on the triangular fossa of the ear

(2) Bergamot on the auricular Point Zero at the helix root

(3) Lavender on LI 4 on the dorsum of the hand between the thumb and index finger

(4) Lavender on LR 3 on the dorsum of the foot between the large and second toes

(5) Atlas cedarwood is placed on KI 3 in the inner leg between the medial malleolus and the Achilles tendon.

All patients were advised to wipe the Bergamot off their ears to prevent photosensitivity or sunburn from sun exposure, and female patients were counseled to avoid pressure on LI 4 if they were pregnant because of a contraindication involving this point in pregnancy.

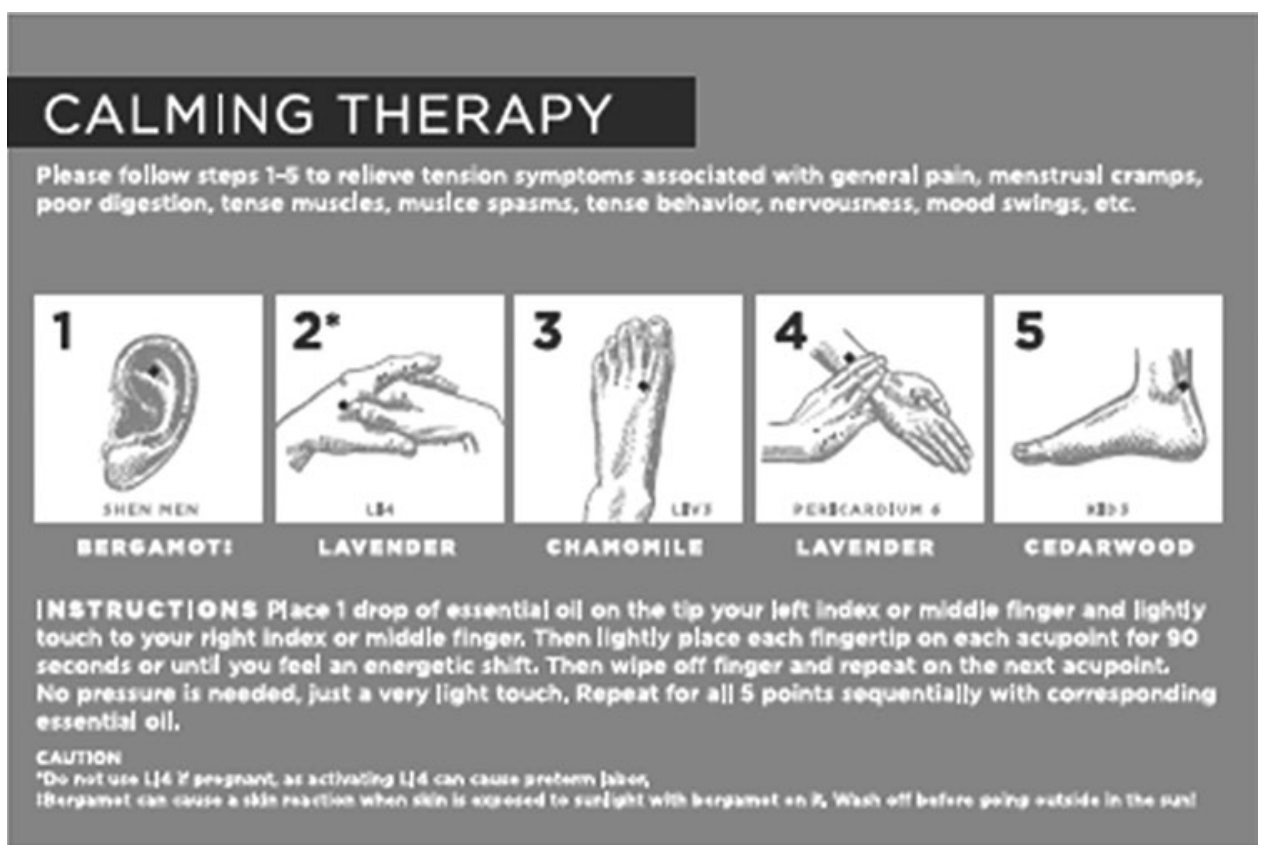

FIG. 1. Aroma Acupoint Therapy "Tense" protocol (also called "Calming Therapy"). Reprinted with permission from Maripossa Wellness. (Latin binomials for the herbs are provided in the text.) 


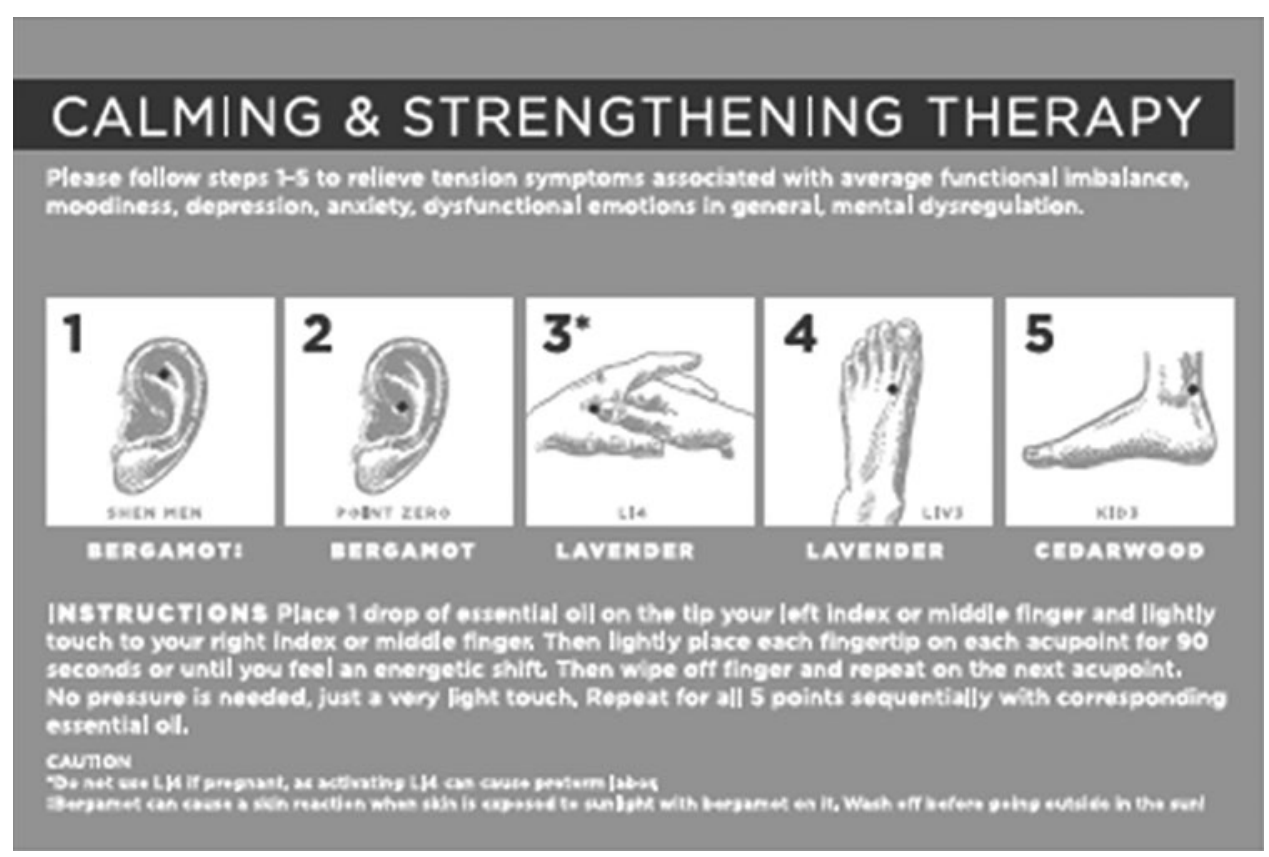

FIG. 2. Aroma Acupoint Therapy "Tense Alternating with Weak" protocol (also called "Calming and Strengthening Therapy"). Reprinted with permission from Maripossa Wellness (Latin binomials for the herbs are provided in the text.)

\section{RESULTS}

In total, 15 adolescents, 14 female and 1 male, were treated with AAT-specific protocols (Table 1). Their ages ranged from 12 to 19 , and they were between the ninth and twelfth grades. Eight patients were treated with the Tense protocol after presenting with symptoms such as headaches, menstrual cramps, nausea, or shortness of breath. Seven patients were treated with the Tense Alternating with Weak protocol after presenting with headaches, shortness of breath, chest pain, back pain, or dizziness. Five patients had positive physical examination findings related to their chief complaints: 2 patients were "tachypnic," 1 patient was "pale and trembling," 1 patient was "crying and hyperventilating," and 1 patient was "tearful." All of these patients had documented resolution of these physical examination findings after receiving AAT.

AAT was used in addition to standard medical therapy. Six patients had taken medications, such as acetaminophen or ibuprofen, prior to their health-center visits. Seven patients were given nonopiate pharmacotherapy in addition to their AAT treatment. One patient was given a heat pack in addition to AAT.

Pain scores before and after treatment were recorded in 8 of 15 encounters. Pain scores were on a 10-point scale with 10 being the highest level of pain and 0 being no pain. The average decrease in pain score was 4 for both treatment protocols. Subjective documentation in all of the encounters suggested that the patients experienced an overall reduction in symptoms, and only 1 patient was unable to return to class after treatment. This patient had a history of migraine headaches and transferred to the emergency department due to an intractable headache. Seven patients were documented in the providers' assessments as having symptoms related to anxiety.

\section{DISCUSSION}

As demonstrated in this case series, AAT is safe and noninvasive, easily taught to a variety of practitioners and well-received by health care providers and patients. The pediatrician and nurse-practitioners who participated reported that the techniques were enjoyable, efficient (it took $\sim 8$ minutes to complete a session) and were easy to learn. The American Academy of Pediatrics has long recognized the need for more resources on integrative medicine, both for health care providers and for patients. In 2008, the Task Force on Complementary and Alternative Medicines released a report emphasizing this need. The Task Force reported that more that $50 \%$ of children with chronic or life limiting illnesses utilized a form of complementary (integrative) or alternative medicine, almost always as an adjunct to standard medical care. ${ }^{2}$ Despite this growing need, there has been little emphasis on research regarding efficacy of these approaches. Additionally, a substantial percentage of complementary and integrative medicine, such as chiropractic and acupuncture, requires numerous hours of additional training and licensure. ${ }^{2}$ The present research demonstrated the potential for adopting AAT as a safe, noninvasive, and effective treatment modality for symptom management in adolescent patients. 


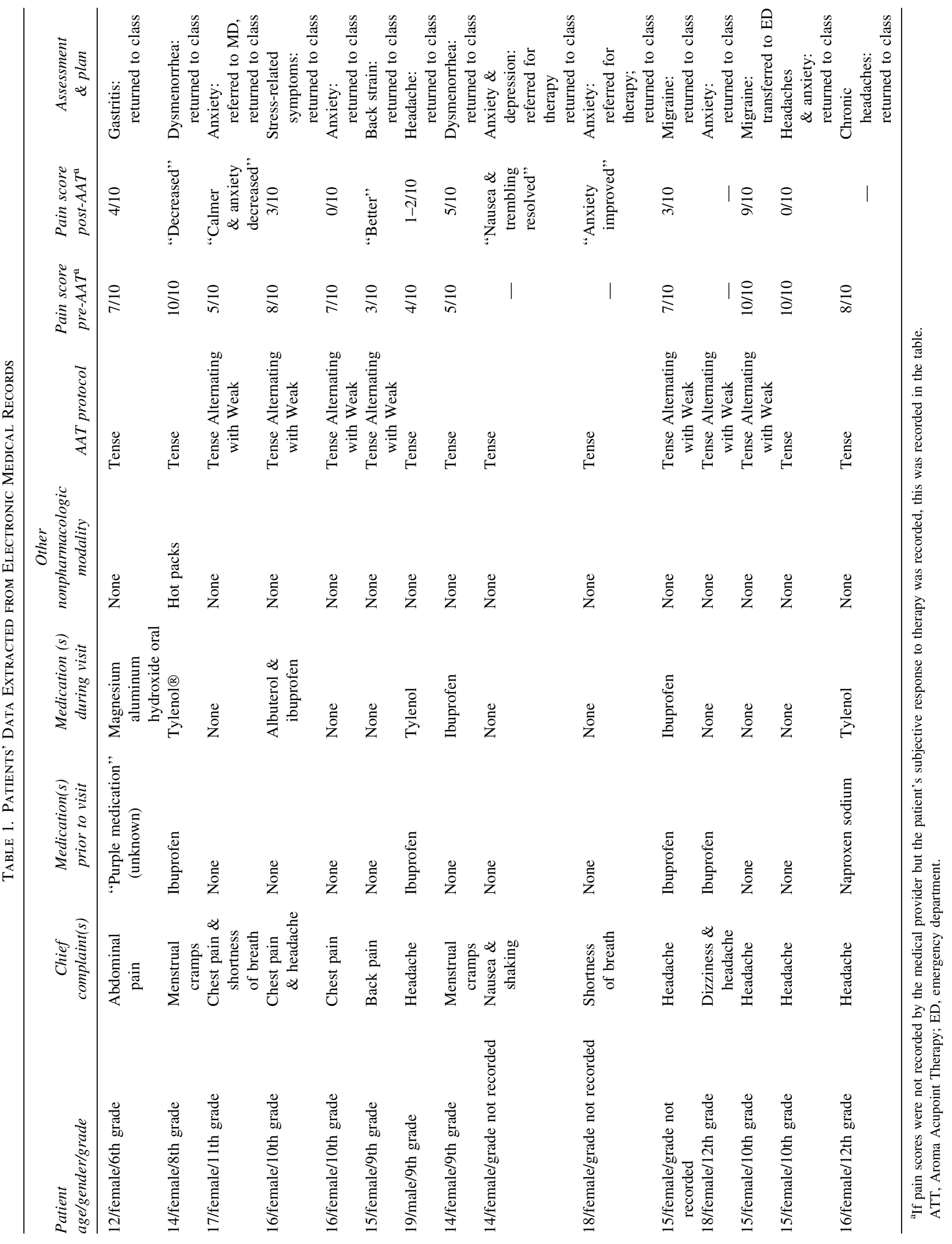




\section{CONCLUSIONS}

This case series introduces AAT as a treatment modality, and opens the door for robust studies of the clinical efficacy of AAT in the form of a clinical trial. Resources, such as electronic medical-record documentation aids, including text expanders, can ensure more uniform documentation, particularly of pre- and post treatment pain scales. Additionally, the efficacy of AAT could be evaluated among specific cohorts of pediatric patients, for example patients presenting with a similar symptom or undergoing the same procedure.

\section{ACKNOWLEDGMENTS}

Many thanks to Karina Muller, MBA, of Maripossa Wellness for working with the authors and the school based health center team to create the Stress Relief Kits containing the 4 essential oils with the accompanying training pamphlets used in this case series. Additional thanks to Janet Garth, MPH, for supporting the expansion of integrative health services at the New York Presbyterian SBHCs.

\section{AUTHOR DISCLOSURE STATEMENT}

No competing financial interests exist.

\section{FUNDING INFORMATION}

No funding was provided for this research.

\section{REFERENCES}

1. Joint Commission. Pain Assessment and Management Standards for Hospitals: R3 Report Issue 11. Online document at: www.jointcommission.org/en/standards/r3-report/r3-reportissue-11-pain-assessment-and-management-standards-forhospitals/ Accessed June 6, 2020.

2. Kemper KJ, Vohra S, Walls R; Task Force on Complementary and Alternative Medicine; Provisional Section on Complementary, Holistic, and Integrative Medicine; American Academy of Pediatrics. The use of complementary and alternative medicine in pediatrics. Pediatrics. 2008;122(6): 1374-1386.

3. McClafferty H, Vohra S, Bailey M, et al. Pediatric integrative medicine. Pediatrics. 2017;140(3):e20171961.

4. Holmes P. Aromatica, vol. 2: A Clinical Guide to Essential Oil Therapeutics. Applications and Profiles. London: Singing Dragon; 2019.
5. Plant RM, Dinh L, Argo S, Shah M. The essentials of essential oils. Adv Pediatr. 2019;66:111-122.

6. Bikmoradi A, Khaleghverdi M, Seddighi I, Moradkhani S, Soltanian A, Cheraghi F. Effect of inhalation aromatherapy with lavender essence on pain associated with intravenous catheter insertion in preschool children: A quasi-experimental study. Complement Ther Clin Pract. 2017;28:85-91.

7. Jafarzadeh M, Arman S, Pour FF. Effect of aromatherapy with orange essential oil on salivary cortisol and pulse rate in children during dental treatment: A randomized controlled clinical trial. Adv Biomed Res. 2013;2:10.

8. Lakhan SE, Sheafer H, Tepper D. The effectiveness of aromatherapy in reducing pain: A systematic review and metaanalysis. Pain Res Treat. 2016;2016:8158693.

9. Marofi M, Sirousfard M, Moeini M, Ghanadi A. Evaluation of the effect of aromatherapy with Rosa damascena Mill. on postoperative pain intensity in hospitalized children in selected hospitals affiliated to Isfahan University of Medical Sciences in 2013: A randomized clinical trial. Iran J Nurs Midwifery Res. 2015;20(2):247-254.

10. Ndao DH, Ladas EJ, Cheng B, et al. Inhalation aromatherapy in children and adolescents undergoing stem cell infusion: Results of a placebo-controlled double-blind trial. Psychooncology. 2012;21(3):247-254.

11. Nord D, Belew J. Effectiveness of the essential oils lavender and ginger in promoting children's comfort in a perianesthesia setting. J Perianesth Nurs. 2009;24(5):307-312.

12. Soltani R, Soheilipour S, Hajhashemi V, Asghari G, Bagheri M, Molavi M. Evaluation of the effect of aromatherapy with lavender essential oil on post-tonsillectomy pain in pediatric patients: A randomized controlled trial. Int J Pediatr Otorhinolaryngol. 2013;77(9):1579-1581.

13. Weaver MS, Robinson J, Wichman C. Aromatherapy improves nausea, pain, and mood for patients receiving pediatric palliative care symptom-based consults: A pilot design trial. Palliat Support Care. 2020;18(2):158-163.

14. Yip YB, Tse SH. An experimental study on the effectiveness of acupressure with aromatic lavender essential oil for subacute, non-specific neck pain in Hong Kong. Complement Ther Clin Pract. 2006;12(1):18-26.

15. Yip YB, Tse SH. The effectiveness of relaxation acupoint stimulation and acupressure with aromatic lavender essential oil for non-specific low back pain in Hong Kong: A randomised controlled trial. Complement Ther Med. 2004;12(1): 28-37.

Address correspondence to: Avital M. Fischer, MD Department of Pediatrics Columbia University Irving Medical Center 622 West 168th St VC-4 New York, NY 10032 USA E-mail: Amf2269@cumc.columbia.edu 